\title{
Grain boundary sliding and migration: Effect of temperature and vacancies
}

\author{
Peter Ballo \\ Department of Physics, Faculty of Electrical Engineering and Information Technology, Slovak University of Technology, Ilkovičova 3, \\ 81219 Bratislava, Slovak Republic \\ Nicholas Kioussis and Gang Lu \\ Department of Physics and Astronomy, California State University Northridge, Northridge, California 91330-8268
}

(Received 6 December 2000; published 20 June 2001)

\begin{abstract}
Monte Carlo simulations combined with the embedded-atom method potential have been employed to investigate the microscopic process of the $\Sigma 5$ tilt grain boundary sliding in aluminum. We have studied the atomic structures and the grain boundary sliding/migration energy profile at elevated temperatures in the absence or presence of vacancies. The annealing temperature is found to play an important role in determining the grain boundary energetics and mobility. Contrary to "static" simulations, the simulated annealing (SA) produces new lower energy states of the complex and low-symmetry grain boundary structure. The vacancy formation energy at the first layer from the interface is found to be significantly lower than that at the other layers and the bulk. On the other hand, the vacancy at the interface has a significantly higher formation energy compared to bulk, in very good agreement with recent $a b$ initio electronic-structure calculations. For both "static" and SA simulations, the grain boundary sliding energy profile is smooth, exhibiting several energy peaks and valleys, where the latter are associated with grain boundary migration. The SA scheme reduces the grain boundary sliding/migration energy barrier by about a factor of 3 and increases the rate of migrations. The distribution of atomic energies helps identify the atoms that play a key role in the grain boundary sliding and migration. The grain boundary sliding energy profile in the presence of a vacancy placed at the first layer is very similar to that of the clean boundary, while the vacancy at the interface increases the grain boundary energy and leads to no migration.
\end{abstract}

DOI: 10.1103/PhysRevB.64.024104

PACS number(s): 61.72.Mm

\section{INTRODUCTION}

It is well established that grain boundaries play important roles in the physical properties of polycrystalline materials, such as diffusion and electrical transport, creep resistance, fracture stress, etc. ${ }^{1}$ For example, grain boundaries provide pathways for diffusion into or within a material that can be orders of magnitude faster than through crystalline regions. $^{2-4}$ There have been suggestions that the vacancy formation energy is lower in the grain boundary or in the surface region than that in the bulk. ${ }^{5}$ However, no experimental evidence is available to approximately quantify the extent of these differences. Grain boundary sliding, i.e., the rigid translation of one grain over another parallel to the boundary interface, is one of the principal mechanisms of plastic flow for polycrystalline materials at intermediate to high temperatures (above $0.4 T_{M}$, where $T_{M}$ is the melting point). ${ }^{6}$ Another process that may occur during the grain boundary sliding is grain boundary migration, which is the motion of the interface in the direction perpendicular to the boundary plane. ${ }^{7}$ Both these processes are important for the grain boundary motion, while other lattice defects, such as substitutional impurities, vacancies, and dislocations, can also affect the grain boundary mobility. ${ }^{8}$

Despite the important role of grain boundaries in material properties, our knowledge at the microscopic level is limited. The direct observation of grain boundary structures is limited by the lack of resolution of experimental techniques such as high-resolution transmission electron microscopy (HRTEM), and by the fact that only fully relaxed structures can be ob- served. With the advent of highly powerful computers, simulations at the atomic level can play an increasingly prominent role as an effective alternative to experimental observations. Computer simulations offer the ability to examine the details at a microscopic scale that cannot be obtained from experiments. The equilibrium structures of grain boundaries have been extensively studied, including more recent studies ${ }^{9}$ of diffusion of vacancies and interstitials in grain boundaries. On the other hand, only very limited research has focused on the atomistic simulation of the grain boundary sliding and migration process. ${ }^{10-14}$ Recently, Molteni et al. carried out ${ }^{15}$ $a b$ initio studies of the atomic structure and the energy barriers for grain boundary sliding in aluminum. However, the vast majority of these atomistic simulations has been concerned with the equilibrium structure at zero temperature using local minimization procedures, such as conjugate gradient and steepest descent, which are not efficient to determine the global energy minimum of the system. The results obtained with these simulations may often be incorrect compared with those obtained by HRTEM. Contrary to "static" simulations, the stimulated annealing (SA) technique ${ }^{16}$ produces new lower-energy states of the complex and lowsymmetry grain boundary structure.

The main purpose of the present work is to apply the SA technique combined with the embedded-atom method (EAM) interatomic potential to investigate the microscopic origin of grain boundary sliding properties. We have studied the structures and the sliding energy profile of the $\Sigma 5$ tilt grain boundary in aluminum in the absence or presence of vacancies at elevated temperatures. The remainder of this 
paper is organized as follows. The computational details, the EAM potential used in the Monte Carlo simulations, and the relaxation process used in the atomic simulations are discussed in Sec. II. In Sec. III A, we discuss the equilibrium structure and energy of the $\Sigma 5$ grain boundary as well as the effect of the vacancy placed at different layers from the interface. In Sec. III B, we examine the sliding and migration properties of the $\Sigma 5$ grain boundary in the presence or absence of vacancies. In Sec. IV, a brief summary and statement of conclusions are presented.

\section{METHODOLOGY}

\section{A. Structural optimization}

The grain boundary energy was determined from the difference between the energy of a supercell containing the grain boundary and the energy of a supercell containing an equal number of atoms in the bulk environment, divided by the total grain boundary area. It is well known that the energy minimization (geometry optimization) represents a very difficult problem even for simple systems. Perhaps the most celebrated minimization technique, introduced by Kirkpatric, Gellat, and Vecchi ${ }^{16}$ is the simulating annealing process. Geman and Geman ${ }^{17}$ demonstrated that if the temperature is reduced slowly enough, conventional SA can in principle find the global minimum. The initial temperature in our simulations was set to $465 \mathrm{~K}$ and the system was then cooled to $28 \mathrm{~K}$. The cooling process was implemented using a stepwise-exponential decrease of temperature involving a total of 155000 steps. For a fixed temperature $T$, the atomic positions were displaced and the change in energy associated with the displacement is either retained or undone using the standard Metropolis technique. ${ }^{18}$ The maximum atomic displacement was adjusted to ensure an acceptance ratio of 0.5 , which gives well-converged statistical averages, with a typical number of about $10^{3}$ Monte Carlo trials for each temperature step.

\section{B. Interatomic potential}

The interatomic potential used in the simulations is based on the EAM, ${ }^{19}$ which provides an accurate description of metallic systems. The EAM has been described elsewhere ${ }^{19,20}$ and so will just be summarized here. Within the EAM, each atom in a solid is viewed as an impurity embedded in a host comprising all the other atoms. The energy for an $n$-atom system then can be written as

$$
E=\sum_{i=1}^{n} E_{i}
$$

where the energy of atom $i$ is given by

$$
E_{i}=\frac{1}{2} \sum_{j(j \neq=i)} \Phi\left(r_{i j}\right)+F\left(\rho_{i}\right) .
$$

Here, $r_{i j}$ is the distance between atoms $i$ and $j, \Phi$ is a pairwise interaction potential, and $\rho_{i}$ is the density at atomic site $i$ due to all of its neighbors (located within a sphere of radius $\left.r_{\text {cutoff }}\right)$ :

$$
\rho_{i}=\sum_{j(j \neq=i)} \rho\left(r_{i j}\right) .
$$

The embedding function $F\left(\rho_{i}\right)$ can be interpreted as the energy arising from embedding atom $i$ in an electron gas of density $\rho_{i}$. The accuracy of the results depends mainly on the approximation of the embedding function and its ability to treat electron density deviations in local coordination. ${ }^{20}$ The EAM potential used in this work was that of Mishin et al. constructed ${ }^{21}$ on a large set of experimental and $a b$ initio databases. More specifically, the experimental part of the database included the following physical properties: the equilibrium lattice parameter, the cohesive energy, the elastic constants $c_{11}, c_{12}$, and $c_{44}$, the vacancy formation energy, the vacancy migration energy, the intrinsic stacking-fault energy, and a few short-wavelength phonon frequencies. Two more experimental properties included in the database (but with lower weight in the fitting procedure) were the surface energy of low-index planes and the equation of state. The $a b$ initio database included the energies of different alternative crystalline structures. On the other hand, the $\Sigma 5$ grain boundary energy was not included in the fitting process and thus it can be used to test the EAM potential with more recent $a b$ initio calculations. ${ }^{22}$ In contrast to other EAM potentials used in the literature, this interatomic potential predicts $^{21}$ that the $\Sigma 5$ (210) grain boundary energy in aluminum is $0.482 \mathrm{~J} / \mathrm{m}^{2}$, in excellent agreement with the $a b$ initio $^{22}$ value of $0.502 \mathrm{~J} / \mathrm{m}^{2}$.

\section{RESULTS AND DISCUSSION}

\section{A. Equilibrium grain boundary structure and energetics}

The tilt $\Sigma 5$ [001] (210) boundary is constructed by means of the coincidence site lattice (CSL) model, in which the lattices of the two grains share one out of five lattice sites. We show in Fig. 1 the relaxed supercell for the $\Sigma 5$ tilt grain boundary employed in the EAM calculations viewed along the [001] direction. The grain boundary is simulated by a 160-atom supercell that consists of 40 (210) atomic layers, corresponding to a separation of $18.46 \AA$ between the two grain boundary planes. There are two atomic planes along the [001] direction. The closed and open circles represent atoms distributed on the first and second (001) planes, respectively. With 20 layers in between, the fictitious grain boundary interaction, introduced by the periodic boundary conditions imposed in the supercell calculations, is small. The two-dimensional lattice constant is set to the experimental value of $4.05 \AA{ }^{21}$

In Fig. 2, we plot the grain boundary energy as a function of the Monte Carlo annealing temperature. The starting atomic configuration for each anneal was taken from the previous one. Tests have indicated that, overall, equilibration of the grain boundary requires about 1000 Monte Carlo steps. Note that the use of SA simulations to anneal the grain boundary structure produces new lower-energy configurations that are different from those obtained using conjugategradient techniques. The extrapolated zero-temperature grain boundary energy is $0.482 \mathrm{~J} / \mathrm{m}^{2}$, smaller than the other EAM value of $0.495 \mathrm{~J} / \mathrm{m}^{2}$ (Ref. 21) and the ab initio value of 


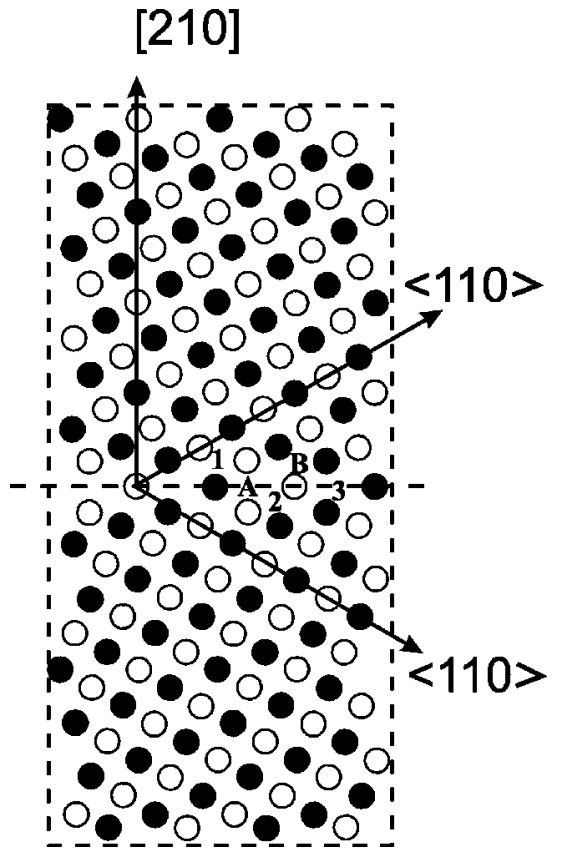

FIG. 1. Relaxed geometry of the 160 -atom supercell for the $\Sigma 5$ tilt grain boundary (210) [001] in aluminum viewed along the [001] direction. The filled and open circles represent atoms on the (001) and (002) planes, respectively.

$0.502 \mathrm{~J} / \mathrm{m}^{2}, 22$ with both latter results obtained from conjugate-gradient techniques. The accuracy of the leastsquares fit of the extrapolated zero-temperature grain boundary energy is $0.003 \mathrm{~J} / \mathrm{m}^{2}$. As will be shown in Sec. III B, the annealing process has a larger effect on the activation energy barriers associated with the grain boundary sliding and migration.

Figure 3(a) shows the relative change of the interlayer spacing normal to the grain boundary plane as a function of the layer number from the interface. One can see a symmet-

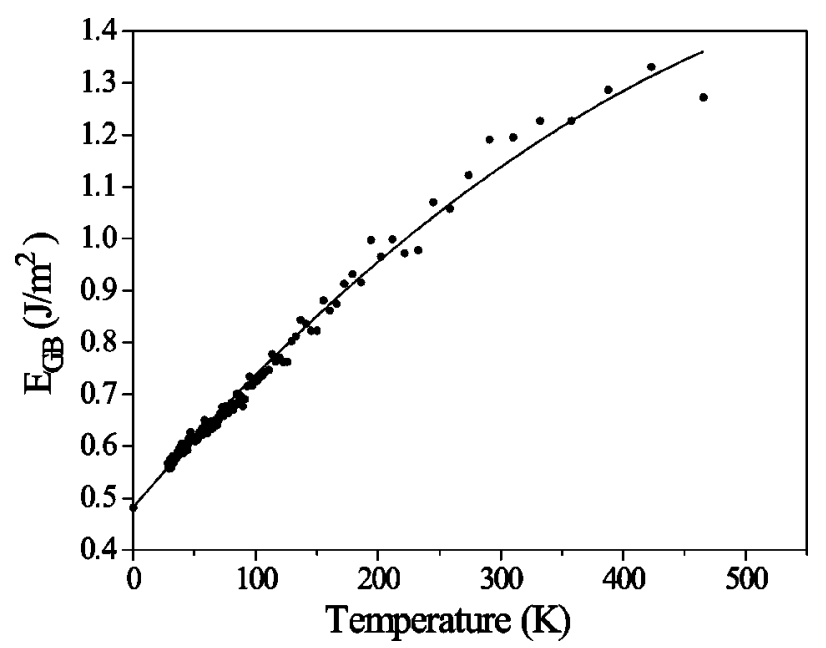

FIG. 2. Grain boundary energy as a function of the Monto Carlo annealing temperature. The solid curve represents the least-squares fit of the Monto Carlo simulations results (circles). The extrapolated zero-temperature grain boundary (GB) energy is $E_{\mathrm{GB}}$ $=0.482 \mathrm{~J} / \mathrm{m}^{2}$.

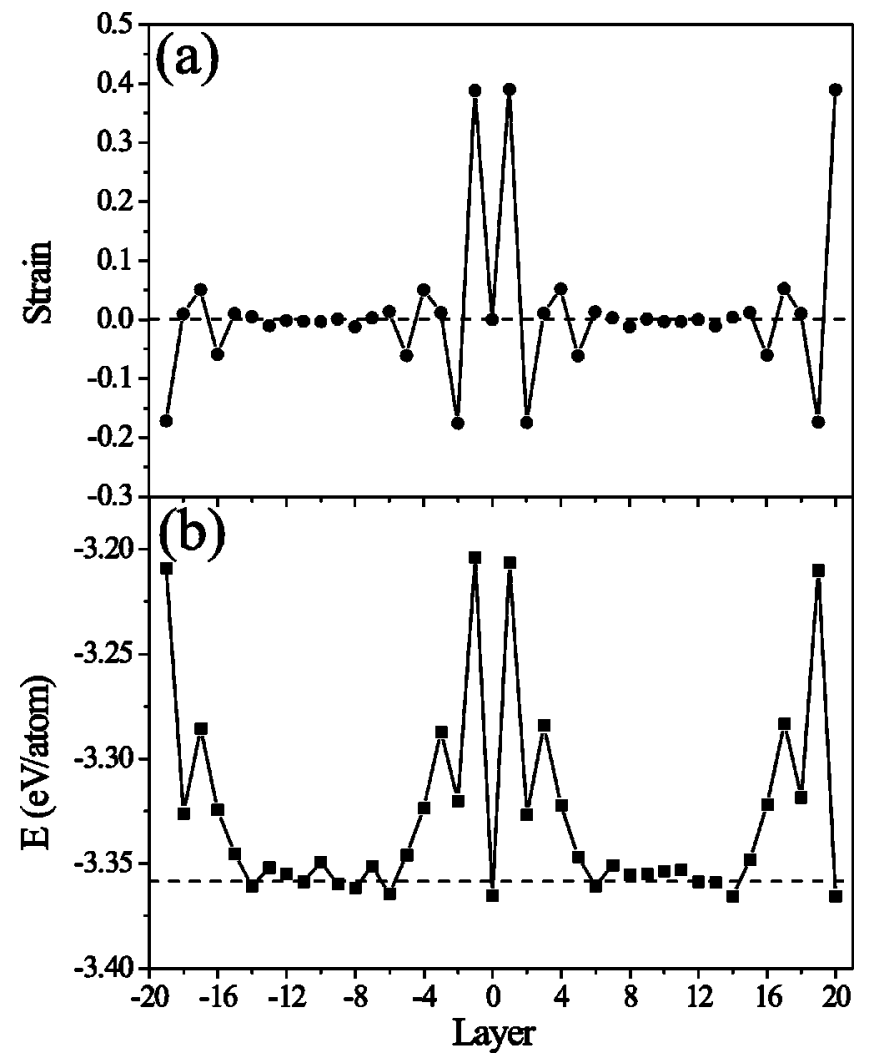

FIG. 3. (a) Relative change of the interlayer spacing normal to the grain boundary and (b) distribution of energy of atoms on different layers as a function of the layer away from the interface. Dashed line denotes the cohesive energy of bulk.

ric oscillation profile of the strain that has a maximum at the boundary plane and decays into the bulk. The small strain of the fourth layer indicates that the atomic relaxation is localized within a few layers from the interface. Figure 3(b) shows the energy profile of the grain boundary atoms as a function of the layer away from the interface. Note that the atoms having an energy higher than the bulk value $(-3.36 \mathrm{eV})$ (Ref. 23) are those within four layers from the interface.

We have also calculated the formation energy for a vacancy placed on various layers from the boundary plane. In Fig. 4 , we show the vacancy formation energy, $E_{v}^{f}$, versus the layer number from the interface. For comparison, the vacancy formation energy in bulk of $0.68 \mathrm{eV}$ (Ref. 24) is also shown by the straight line. It is clear that the vacancy formation energy at the first layer is significantly lower than that at other layers or bulk, while the vacancy at the interface has larger formation energy compared to the bulk value. These results, which are in good agreement with those of our recent $a b$ initio electronic-structure calculations, ${ }^{22}$ suggest that while the first layer acts as a sink for vacancies, the grain boundary plane does not favor the formation of vacancies. The vacancy formation energy at the seventh layer from the interface is close to the bulk value, showing the convergence of the energy with respect to the supercell size.

In order to understand the variation of the vacancy formation energy, we show in Fig. 5 the atomic configurations for 


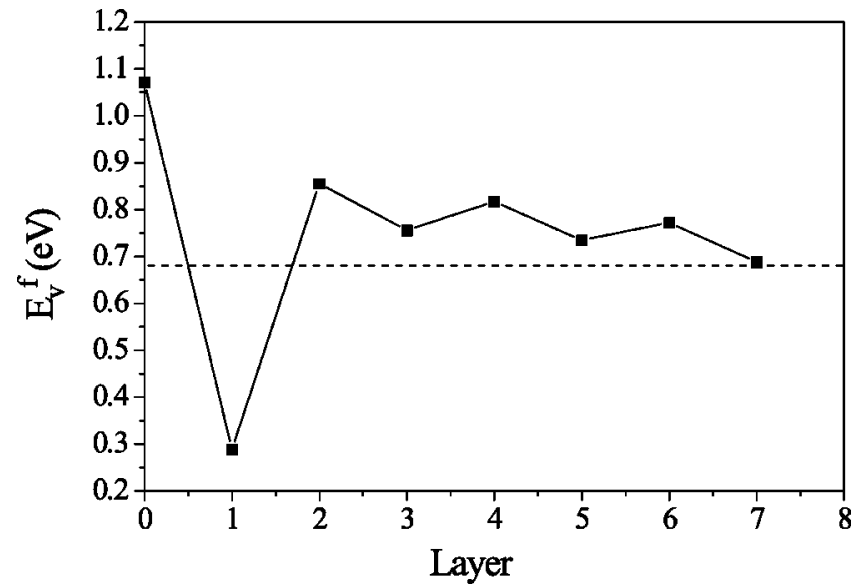

FIG. 4. Vacancy formation energy $E_{v}^{f}$ versus the layer number from the interface. The dashed line denotes the vacancy formation energy for bulk.

the perfect grain boundary [Fig. 5(a)], the grain boundary with the vacancy placed at the interface [Fig. 5(b)], and the grain boundary with the vacancy placed in the first layer from the interface [Fig. 5(c)]. The atoms are labeled with a different level of gray, which represents the energy $E_{i}$ [Eq. (2)] of each atom in the boundary region according to the energy scale (in eV) panel on the right. As with any symmetric tilt grain boundary, all atoms, except for those on the interface, have a twin across the interface. The nearestneighbor twin atoms across the interface have higher energies because their distance of $2.56 \AA$ is shorter than that of $2.86 \AA$ in bulk. On the other hand, the atom at the grain boundary plane has a lower energy due to the absence of a twin atom. Note, while more distant neighbors should also play a role in the energetics, their contribution turns out to be rather small (typically less than $10 \%$ of the nearest-neighbor contribution $^{25}$ ).

If we remove an atom from the grain boundary plane (creating a vacancy) in Fig. 5(b), the relaxation of the nearest-neighbor shell (symmetrically placed around the vacancy) causes no lateral forces and hence very weak atomic relaxation around the vacancy even at elevated temperatures. The increase in the vacancy formation energy relative to the bulk value is associated with the increase in energy (about $0.3 \mathrm{eV} / \mathrm{atom})$ of the nearest-neighbor twins across the interface. The creation of a vacancy at the first layer from the interface [Fig. 5(c)] breaks the reflection symmetry in the grain boundary region with the remaining twin atoms showing a tending to fill the empty space. The atoms relax essentially perpendicularly towards the interface with a maximum displacement of $0.56 \AA$. This large structural relaxation reduces the vacancy formation energy by $58 \%$ of its bulk value. As shown in Fig. 5(c), there is an overall reduction of the atomic energies compared to the corresponding values in the clean grain boundary [Fig. 5(a)]. It should be pointed out that this grain boundary structure turns out to be very stable even at elevated temperatures because the vacancy cannot migrate from the grain boundary to the bulk region, due to a

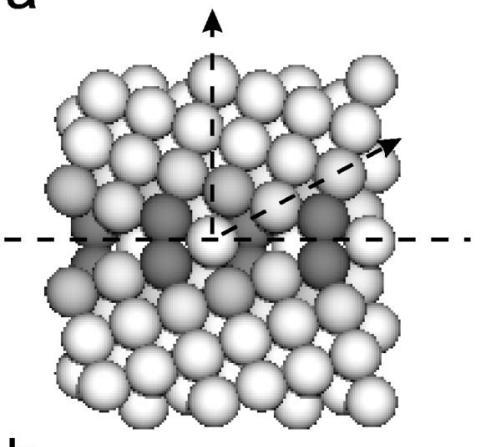

$-3.13$

$-3.16$

$-3.19$

$-3.22$

$-3.25$

$-3.28$

$-3.31$

$-3.34$

b

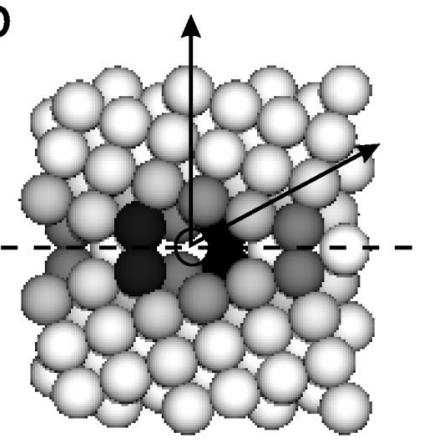

$-3.37$

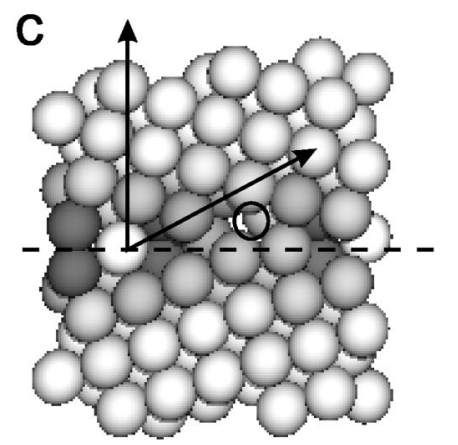

FIG. 5. Energy distribution of atoms in the grain boundary for the (a) clean grain boundary, (b) vacancy placed in the boundary plane, and (c) vacancy placed in the first layer. The level of gray indicates the energy of individual atoms according to the energy scale panel in the right top corner in $\mathrm{eV}$.

the high-energy barrier separating the vacancy at the first layer from the rest of the grain boundary region.

\section{B. Grain boundary sliding and migration}

The grain boundary sliding is simulated quasistatically, by rigidly shifting the top grain with respect to the bottom by a small specified distance along the $[1 \overline{2} 0]$ direction. At each displacement, the system is then relaxed using Monte Carlo simulations. In order to investigate the effect of annealing on the boundary sliding process, such as the energy barriers and the shear displacements at which the grain boundary migration occurs, we have carried out two sets of Monte Carlo simulations. In the first, which we will refer to as "static," the probability acceptance of any higher-energy configurations is set equal to zero, thus driving the system to the local minimum configuration. In the second set of simulations, the 


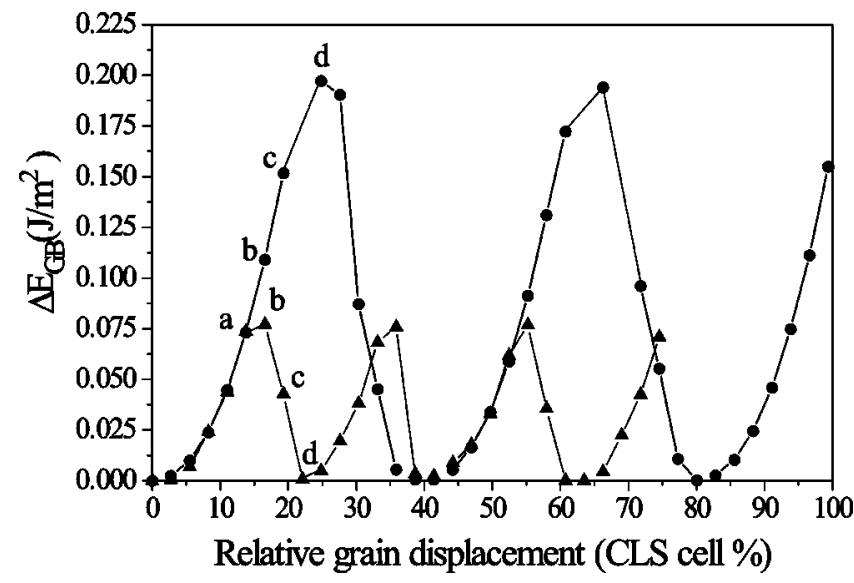

FIG. 6. The variation of the relative grain boundary energy during sliding as a function of the sliding distance expressed as a percentage of the lattice parameter, $a_{\mathrm{CSL}}$, of the CSL cell along [120]. The circles and triangles correspond to the "static" and the SA simulations, respectively. The labels from (a)-(d) represent the special atomic configurations displayed in Figs. 7 and 8.

probability acceptance follows standard Metropolis rules by lowering gradually the temperature from $465 \mathrm{~K}$ to $28 \mathrm{~K}$.

In Fig. 6, we show the relative variation of the grain boundary energy (with respect to its zero displacement value) as a function of the grain boundary sliding displacement. The sliding distance is expressed in a percentage of $a_{\mathrm{CSL}}$, where $a_{\mathrm{CSL}}$ is the lattice parameter of the CSL cell along [120]. We plot the results for the energies for both the "static" (circles) and SA (triangles) calculations. The labels (a) -(d) on both curves indicate the special atomic configurations, which will be explored in detail below. For both cases, the energy profile is smooth, exhibiting several energy peaks and valleys between them, with the latter being associated with the grain boundary migration. It is important to notice that the SA approach reduces the grain boundary sliding energy barrier by about a factor of 3 compared to the corresponding "static" values, and it also increases the frequency of grain boundary migrations. The migrations in the SA simulations occur earlier and hence are more frequent during sliding. For example, the first migration occurs when the shear displacement is about $22 \% a_{\mathrm{CSL}}$, compared to the corresponding value of $40 \% a_{\mathrm{CSL}}$ in the "static" calculation. These results indicate that, contrary to "static" simulations, the SA approach helps the system escape from the local energy minimum configurations, thus producing new lowerenergy states of the complex and low-symmetry grain boundary structure.

In Figs. 7 and 8, we display the atomic configurations associated with the points (a)-(d) in the energy curves in Fig. 6, for the "static" and SA simulations, respectively. As in Fig. 5, the atoms are labeled with a different level of gray, which represents the energy $E_{i}$ of each atom in the grain boundary according to the energy scale (in $\mathrm{eV}$ ) panel on the right. The atoms that control the energetics of the grain boundary sliding are labeled in Fig. 1 by 1, 2, and 3 for one of the (001) planes and by $A$ and $B$ for the other one. The sliding process breaks the symmetric arrangement of the ini- a

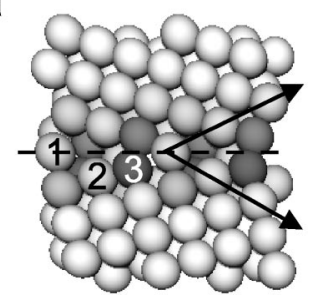

C

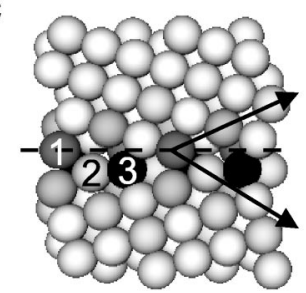

b

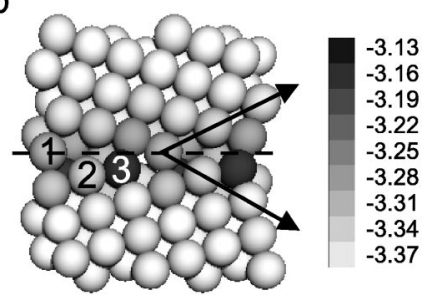

d

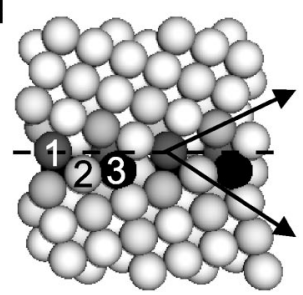

FIG. 7. Evolution of the atomic structure of the pure grain boundary during the sliding process using "static" simulations. The panels (a)-(d) correspond to the special points on the "static" energy curve in Fig. 6.

tial equilibrium grain boundary structure. During the sliding process, atoms across the interface change their relative distances and the original twin atoms no longer face each other. Atoms 1 and $B$ lying in the top grain are pushed toward atoms $A$ and 3 in the bottom grain, and thus their energy increases. For the "static" calculation, the energy of individual atoms increases from (a) to (d) in Fig. 7. Note that the energy of the twin atom of 3 decreases while its distance across the grain boundary increases so as to maintain the optimal bulk coordination. The increase in separation between atom 2 and its twin across the interface (caused by the sliding of the upper grain) results in the formation of empty space in the first layer of the upper grain, which can potentially be filled by atom 2 in the lower grain. However, this process is associated with an energy barrier that is too high to overcome for the static simulations. Therefore, in the a

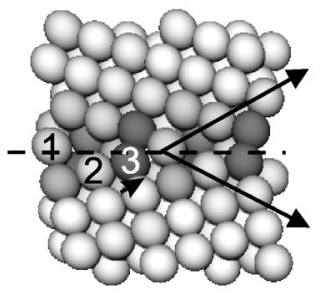

c

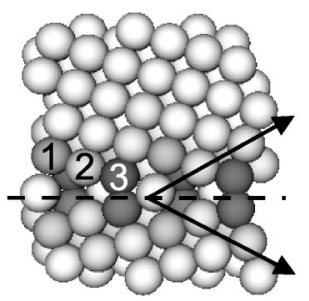

b
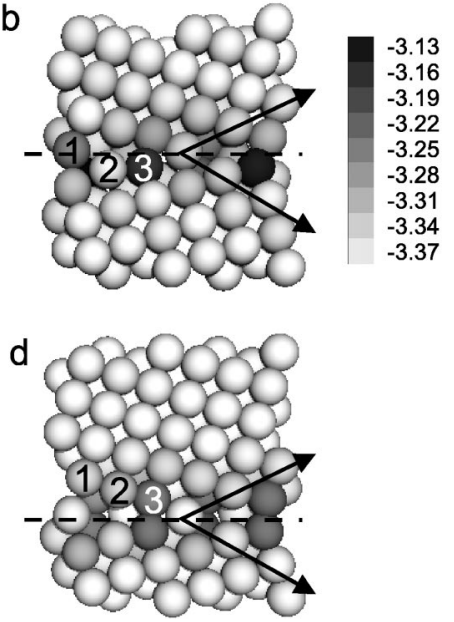

FIG. 8. Evolution of the atomic structure of the pure grain boundary during the sliding process using SA simulations. The panels (a)-(d) correspond to the special points on the SA energy curve in Fig. 6. 


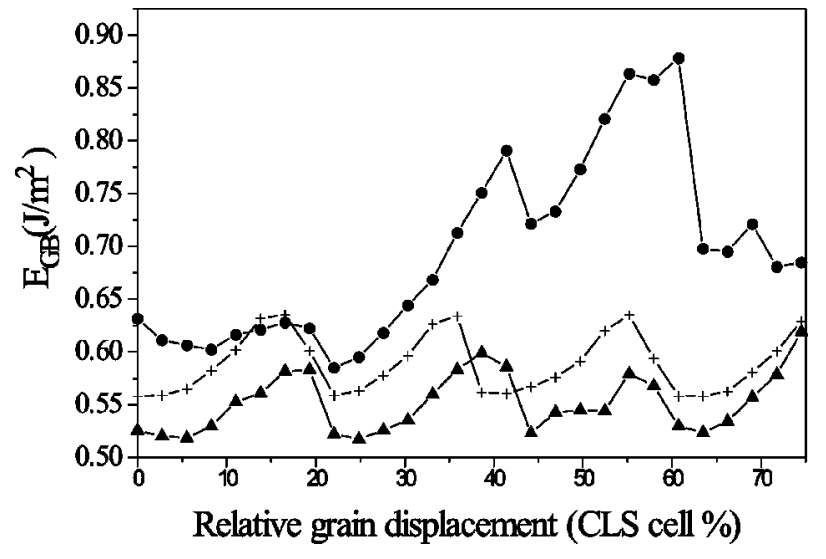

FIG. 9. Variation of the grain boundary energy in the presence of a vacancy as a function of the sliding distance expressed as a percentage of the lattice parameter, $a_{\mathrm{CSL}}$, of the CSL cell along [120]. The squares and circles denote the vacancy in the boundary plane and the first layer, respectively, while the crosses denote the clean grain boundary.

"static" calculations, atom 2 is pinned during the sliding stages (a)-(d) in Fig. 7, and hence the grain boundary energy increases. On the other hand, the SA scheme allows atom 2 to overcome the energy barrier and to move into the empty space in the upper grain with a relaxation of $0.45 \AA$ [Figs. $8(\mathrm{c})$ and $8(\mathrm{~d})]$. Hence, it is the ability of atom 2 to escape from local minima and to relax into the upper grain, which is crucial for the grain boundary migration. A surprisingly small relaxation of atom 3 in the upper grain gives rise to the grain boundary migration and hence decreases the grain boundary energy. One can infer from these calculations that in principle rapid grain boundary migrations are possible due to this orchestrated atomic relaxation involving only a small number of atoms.

In Fig. 9, we plot the variation of the grain boundary energy (calculated from the SA scheme) as a function of the grain boundary sliding distance for a vacancy placed at the grain boundary plane (circles) and the first plane from the interface (triangles). We have removed atom 1 or atom $A$, respectively. As a reference, we also plot the variation of the energy for the vacancy-free (clean) grain boundary (crosses). As expected, the sliding and migration process is considerably more complicated in the presence of vacancies. Note that for both vacancy positions, the grain boundary energy initially decreases for small sliding distances. This arises from the fact that the short-range repulsion between the nearest-neighbor twins across the boundary plane decreases. Thus, the vacancy configurations in Figs. 5(b) and 5(c) for zero sliding represent local metastable equilibrium configurations. This is consistent with results of high resolution transmission microscopy experiments, ${ }^{26}$ which show partial atomic rearrangement after a short lateral sliding, and with our recent $a b$ initio electronic-structure calculations showing that the shifted clean tilt grain boundary has lower energy than the "symmetric"' tilt CSL model. However, in general, there is no guarantee that the shifted tilt grain boundary has the lowest energy because the boundary is based on the CSL model, which does not allow rotational degrees of freedom for the grain boundary. Therefore the equilibrium structure obtained here is only correct within the context of the symmetric tilt CSL grain boundary model. Overall, these results indicate that in finding equilibrium structures of grain boundaries, small lateral shifts should also be checked. Furthermore, the difference in energy between the equilibrium structure for the vacancy in the grain boundary plane and in the first neighboring plane at about $10 \%$ slide is about $0.58 \mathrm{eV}$ compared to the corresponding value of $0.8 \mathrm{eV}$ at $0 \%$ shear (Fig. 4). However, even though the effect of vacancy trapping is reduced by about $20 \%$, the effect remains clear. For the case of the vacancy placed at the first layer, the variation of the grain boundary energy is very similar to that of the clean grain boundary and is accompanied by migrations. The absence of an atom in the first layer eliminates the shortrange repulsion between the nearest-neighbor twin atoms across the grain boundary. This in turn changes the grain boundary migration process, in which the vacancy at the first layer does not affect the relaxation of atom 2, which is the pivotal atom for the migration. On the other hand, a vacancy placed at the boundary results in an increase of the energy of the nearest-neighbor twins across the boundary [see Fig. $5(\mathrm{~b})$. Moreover, the structural relaxation during sliding is weak since the atoms at the boundary have no twins. Thus, the vacancy destroys the coupling between sliding and migration and the grain boundary energy increases.

\section{CONCLUSIONS}

We have employed Monte Carlo simulations combined with the EAM potential to investigate the microscopic process of grain boundary sliding in aluminum. Contrary to "static" simulations, the SA produces new lower-energy states of the complex and low-symmetry grain boundary structure. We have studied the atomic structures and the sliding energy profile in the absence or presence of a vacancy at elevated temperatures. It is found that the annealing temperature plays an important role in determining the grain boundary energetics and the grain boundary mobility.

The vacancy formation energy for the first layer from the interface is found to be significantly lower compared to that for all other layers and also to the bulk value, while a vacancy at the interface has significantly higher formation energy compared to bulk. This indicates that while the first layer acts as sinks of vacancies, the grain boundary interface itself does not favor the formation of vacancies. These results agree with our recent $a b$ initio calculations. ${ }^{22}$ For both "static" and SA simulations, the grain boundary sliding energy profile is smooth, exhibiting several energy peaks and valleys, while the latter is found to be associated with grain boundary migrations. The SA approach reduces the grain boundary sliding/migration energy barrier by about a factor of 3 and therefore increases the rate of migrations. Analysis of the atomic energies in the grain boundary region provides insight into the relevant physical process and unveils the atomic mechanism for the coupling of grain boundary sliding and migration. The grain boundary sliding energy profile in 
the presence of a vacancy at the first plane is very similar to that of the clean grain boundary and is accompanied with grain boundary migrations. On the other hand, a vacancy placed at the grain boundary plane increases the grain boundary energy during the sliding and leads to no migration.

\section{ACKNOWLEDGMENTS}

This research was supported through the North Atlantic Treaty Organization under Grant No. HTECH.LG.970619 and the U.S. Army under Grant No. DAAD19-00-1-0049.
${ }^{1}$ A. P. Sutton and R. W. Balluffi, Interface in Crystalline Materials (Oxford University Press, Oxford, 1995).

${ }^{2}$ I. Kaur, Y. Mishin, and W. Gust, Fundamentals of Grain and Interphase Boundary Diffusion (John Wiley and Sons, Sussex, 1995).

${ }^{3}$ Y. Mishin and C. Herzig, Mater. Sci. Eng., A 260, 55 (1999).

${ }^{4}$ J. Bernardini, S. Benis, and G. Moya, Defect Diffus. Forum 6669, 805 (1989).

${ }^{5}$ R. W. Balluffi, in Diffusion in Crystalline Solids, edited by G. E. Murch and A. S. Nowick (Academic Press, New York, 1984), p. 320.

${ }^{6}$ R. Z. Valiev, V. G. Khairullin, and A. D. Sheikh-Ali, in Structure and Property Relationships for Interfaces, edited by J. L. Walter, A. H. King, and K. Tangri (ASM International, Metals Park, OH, 1991), p. 309.

${ }^{7}$ D. A. Smith, in Materials Interfaces, Atomic-level Structure and Properties, edited by D. Wolf and S. Yip (Cambridge University Press, Cambridge, 1992), p. 212.

${ }^{8}$ C. H. Wörner and A. Cabo, Acta Metall. 35, 2801 (1987).

${ }^{9}$ M. R. Sorensen, Y. Mishin, and A. F. Voter, Phys. Rev. B 62, 3658 (2000).

${ }^{10}$ G. H. Bishop, R. J. Harrison, T. Kwok, and S. Yip, J. Appl. Phys. 53, 5609 (1982).

${ }^{11}$ Y. Mishin and D. Farkas, Philos. Mag. A 78, 29 (1998).

${ }^{12}$ R. G. Hoagland and M. I. Baskes, Scr. Mater. 39, 417 (1998).

${ }^{13}$ N. Chandra and P. Dang, J. Mater. Sci. 34, 655 (1999).

${ }^{14}$ R. J. Kurz, R. G. Hoagland, and J. P. Hirth, Philos. Mag. A 79,
665 (1999).

${ }^{15}$ C. Molteni, N. Marzari, M. C. Payne, and V. Heine, Phys. Rev. Lett. 79, 869 (1997).

${ }^{16}$ S. Kirkpatrick, C. D. Gellat, and M. P. Vecchi, Science 220, 671 (1983).

${ }^{17}$ S. Geman and D. Geman, IEEE Trans. Pattern Anal. Mach. Intell. PAMI-6, 721 (1984).

${ }^{18}$ M. P. Allen and D. J. Tildesley, Computer Simulation of Liquids (Oxford University Press, Oxford 1996).

${ }^{19}$ M. S. Daw and M. I. Baskes, Phys. Rev. B 29, 6443 (1984).

${ }^{20}$ S. M. Foiles, M. I. Baskes, and M. S. Daw, Phys. Rev. B 33, 7983 (1986).

${ }^{21}$ Y. Mishin, D. Farkas, M. J. Mehl, and D. A. Papaconstantopoulos, Phys. Rev. B 59, 3393 (1999).

${ }^{22}$ G. Lu, N. Kioussis, and V. V. Bulatov, Phys. Rev. B 62, 3099 (2000).

${ }^{23}$ Handbook of Chemistry and Physics, edited by R. C. Weast (CRC, Boca Raton, FL, 1984).

${ }^{24}$ H.-E. Schaefer, R. Gugelmaier, M. Schmoltz, and A. Seeger, Mater. Sci. Forum 15-18, 111 (1987).

${ }^{25}$ S. B. Newcomb, D. J. Smith, and W. M. Stobbs, J. Microsc. 130, 137 (1983)

${ }^{26}$ D. L. Medlin, M. J. Mills, W. M. Stobbs, M. S. Daw, and F. Cosandey, in Atomic-Scale Imaging of Surfaces and Interfaces, edited by D. K. Biegelson, D. J. Smith, and D. S. Y. Tong Mater. Res. Soc. Symp. Proc. No. 295 (MRS, Pittsburgh, 1993), p. 91. 